\title{
On three dimensional quasi-Sasakian manifolds
}

\author{
Nandan Ghosh ${ }^{1}$ and Manjusha Tarafdar ${ }^{2}$ \\ ${ }^{1}$ Department of Mathematics, Asutosh College, Kolkata - 700019, India \\ ${ }^{2}$ Department of Pure Mathematics, University of Calcutta, Kolkata - 700019, India \\ E-mail: ${ }^{1}$ ghosh1989nandanmath@gmail.com, ${ }^{2}$ drmanjushamajumdar@gmail.com
}

\begin{abstract}
Let $\mathrm{M}$ be a 3-dimensional quasi-Sasakian manifold. Olszak [6] proved that $\mathrm{M}$ is conformally flat with constant scalar curvature and hence its structure function $\beta$ is constant. We have shown that in such M, a second order symmetric parallel tensor is a constant multiple of the associated metric tensor. A necessary and sufficient condition for such a manifold to be minimal has been obtained. Finally if such $\mathrm{M}$ satisfies $\mathrm{R}(\mathrm{X}, \mathrm{Y}) . \mathrm{S}=0$, then, $\mathrm{S}$ has two different non-zero eigen values.
\end{abstract}

2010 Mathematics Subject Classification. 53C25. 53C15

Keywords. Quasi-Sasakian manifold, Eigen values.

\section{Introduction}

In 1926, Levi [4] proved that a second order symmetric parallel non singular tensor on a space of constant curvature is a constant multiple of the metric tensor. Sharma [7] generalised Levi's result. In this paper, we have considered a 3-dimensional quasi-Sasakain manifold. Olszak [6] proved that such a space is conformally flat with constant scalar curvature and hence the structure function $\beta$ is constant. In this paper we have shown that in a 3-dimensional quasi- Sasakian manifold with constant scalar curvature, a second order symmetric parallel tensor is a constant multiple of the associated metric tensor. In the next section, we consider 3-dimensional quasi-Sasakian manifold with constant scalar curvature which are hypersurfaces of a Riemannian manifold of constant curvature 1. A necessary and sufficent condition for such a manifold to be minimal has been obtained. Lastly,if a three dimensional quasi-Sasakian manifold with constant scalar curvature satisfies $R(X, Y) . S=0$, then it is proved that the symmetric endomorphism $Q$ of the tangent space corresponding to $\mathrm{S}$ has two different non-zero eigen values. Let $\mathrm{M}$ be an almost contact metric manifold of dimension $(2 \mathrm{n}+1)$ with an almost contact metric structure $(\varphi, \xi, \eta, \mathrm{g})[2]$ where $\varphi, \xi, \eta$ are tensor fields of type $(1,1),(1,0),(0,1)$ respectively and $\mathrm{g}$ is a Riemannian metric on $\mathrm{M}$ such that

$$
\begin{aligned}
& \varphi^{2}=-I+\eta \otimes \xi, \eta(\xi)=1, \varphi \xi=0, \eta \circ \varphi=0, \eta(X)=g(X, \xi), \\
& g(\varphi X, \varphi Y)=g(X, Y)-\eta(X) \eta(Y)
\end{aligned}
$$

$\forall \mathrm{X}, \mathrm{Y} \in \mathrm{TM}$.

$\mathrm{M}$ is said to be quasi-Sasakian, if it is normal and the fundamenatal 2-form $\Phi$ is closed $(d \Phi=0$, $\Phi(X, Y)=g(X, \varphi Y)[1])$. It has been proved [5] that an almost contact metric manifold $\mathrm{M}$ of dimension 3 is quasi-Sasakian if and only if

$$
\nabla_{X} \xi=-\beta \varphi X
$$

Tbilisi Mathematical Journal 9(1) (2016), pp. 23-28.

Tbilisi Centre for Mathematical Sciences.

Received by the editors: 17 July 2015.

Accepted for publication: 18 December 2015. 
for a certain function $\beta$ on $M$ such that $\xi \beta=0$. Hence

$$
\left(\nabla_{X} \varphi\right) Y=\beta(g(X, Y) \xi-\eta(Y) X) .
$$

Now by Theorem 3.6 of [6], such a space is conformally flat with constant scalar curvature. Consequently, if R,S denote the curvature tensor and the Ricci tensor of M, then

$$
\begin{gathered}
R(X, Y) \xi=\beta^{2}(\eta(Y) X-\eta(X) Y) \\
S(X, Y)=\left(\frac{r}{2}-\beta^{2}\right) g(X, Y)+\left(3 \beta^{2}-\frac{r}{2}\right) \eta(X) \eta(Y)
\end{gathered}
$$

$\mathrm{S}(\mathrm{X}, \mathrm{Y})=\mathrm{g}(\mathrm{QX}, \mathrm{Y})$ where $\mathrm{Q}$ is the symmetric endomorphism of the tangent space of $\mathrm{M}$.

$$
\begin{gathered}
S(\varphi X, \varphi Y)=S(X, Y)-2 \beta^{2} \eta(X) \eta(Y) \\
S(X, \xi)=2 \beta^{2} \eta(X) \\
R(\xi, X) \xi=\beta^{2}(\eta(X) \xi-X) .
\end{gathered}
$$

The above results will be used in the next sections.

\section{3-dimensional quasi-Sasakian manifolds with second order symmetric parallel tensor}

Let $\mathrm{T}$ denote a $(0,2)$ tensor field on a 3 -dimensional quasi-Sasakian manifold such that $\nabla \mathrm{T}=0$. Then

$$
T(R(W, X) Y, Z)+T(Y, R(W, X) Z)=0
$$

for arbitrary vector fields $\mathrm{X}, \mathrm{Y}, \mathrm{Z}, \mathrm{W}$ on $\mathrm{M}$.

Taking $\mathrm{Y}=\mathrm{Z}=\mathrm{W}=\xi$ in 1.9 ) we get

$$
T(R(\xi, X) \xi, \xi)+T(\xi, R(\xi, X) \xi)=0 .
$$

Using 1.8) in 1.10) we have

$$
g(X, \xi) T(\xi, \xi)-T(X, \xi)=0
$$

as $\mathrm{T}$ is symmetric. Differentiating 1.11) along $\mathrm{Y}$, we get

$$
\begin{gathered}
\left\{g\left(\nabla_{Y} X, \xi\right)+g\left(X, \nabla_{Y} \xi\right)\right\} T(\xi, \xi)+2 g(X, \xi) T\left(\nabla_{Y} \xi, \xi\right) \\
-T(\nabla X, \xi)-T\left(\nabla_{Y} \xi, X\right)=0 .
\end{gathered}
$$

as $\mathrm{T}$ is symmetric.

Putting $X=\nabla_{Y} X$ in 1.11) we find

$$
g\left(\nabla_{Y} X, \xi\right) T(\xi, \xi)-T\left(\nabla_{Y} X, \xi\right)=0 .
$$

Using 1.12) in 1.13) we find, on using 1.2)

$$
g(X, \varphi Y) T(\xi, \xi)+2 g(X, \xi) T(\varphi Y, \xi)-T(\varphi Y, X)=0 .
$$


Replacing $\mathrm{X}$ by $\varphi \mathrm{Y}$ in 1.11) we find, on using 1.1)

$$
T(\varphi Y, \xi)=0 .
$$

From 1.14) and 1.15) we obtain

$$
g(X, \varphi Y) T(\xi, \xi)-T(\varphi Y, X)=0 .
$$

Replacing $\mathrm{Y}$ by $\varphi \mathrm{Y}$ and using 1.1) and 1.11) we obtain

$$
T(X, Y)=T(\xi, \xi) g(X, Y) .
$$

The fact that $\mathrm{T}(\xi, \xi)$ is a constant can be checked by differentiating it along any vector field on M. Thus we state

Theorem 2.1. On a 3-dimensional quasi-Sasakian manifold with constant scalar curvature, a second order symmetric parallel tensor is a constant multiple of the associated metric tensor.

\section{3-dimensional quasi-Sasakian manifolds which are hypersurfaces of a Riemannian manifold of constant curvature}

Let $\mathrm{M}$ be a 3-dimensional quasi-Sasakain manifolds which is isometrically immersed in a Riemannian manifold of dimension 4 of constant curvature 1 . Then we have the Gauss and Coddazi equations $[3]$

$$
\begin{gathered}
R(X, Y)=X \wedge Y+A X \wedge A Y \\
R(X, Y) Z=g(Y, Z) X-g(X, Z) Y+g(A Y, Z) A X-g(A X, Z) A Y \\
\left(\nabla_{X} A\right) Y=\left(\nabla_{Y} A\right) X
\end{gathered}
$$

where $\mathrm{A}$ is a $(1,1)$ tensor field associated with the second fundamental form $\mathrm{B}$ by $\mathrm{B}(\mathrm{X}, \mathrm{Y})=\mathrm{g}(\mathrm{X}, \mathrm{AY})$. Here $\mathrm{A}$ is symmetric with respect to $\mathrm{g}$ and when the trace of $\mathrm{A}$ vanishes, the immersion is called minimal. The rank of $\mathrm{A}$ is called the type number of immersion. Since the Ricci curvature tensor $\mathrm{S}$ is given by

$$
S(X, Y) \rightarrow \operatorname{trace}[W \rightarrow R(X, W) Y]
$$

By 1.19) we have

$$
S(X, Y)=(3-1) g(X, Y)+(\operatorname{trace} A) g(A X, Y)-g(A A X, Y) .
$$

Replacing $\mathrm{X}$ and $\mathrm{Y}$ by $\varphi \mathrm{X}$ and $\varphi \mathrm{Y}$ in 1.21), we find

$$
S(\varphi X, \varphi Y)=2 g(\varphi X, \varphi Y)+\theta g(A \varphi X, \varphi Y)-g(A A \varphi X, \varphi Y)
$$

where $\theta$ is the trace of A. Again,

$$
\begin{gathered}
g(A \varphi X, \varphi Y)=-g(\varphi A \varphi X, Y), \\
g(A A \varphi X, \varphi Y)=-g(\varphi A A \varphi X, Y) .
\end{gathered}
$$


Using 1.1), and 1.6), it follows from 1.22)

$$
\begin{aligned}
S(X, Y)= & 2 g(X, Y)+2\left(\beta^{2}-1\right) \eta(X) \eta(Y) \\
& -\theta g(\varphi A \varphi X, Y)+g(\varphi A A \varphi X, Y) .
\end{aligned}
$$

Now 1.21) and 1.23) imply

$$
\theta A X-A A X+2\left(1-\beta^{2}\right) \eta(X) \xi+\theta \varphi A \varphi X-\varphi A A \varphi X=0 .
$$

Now if trace of A vanishes, then from 1.24) we get

$$
2\left(1-\beta^{2}\right) \eta(X) \xi=A A X+\varphi A A \varphi X .
$$

If 1.25) holds, then from 1.24), $\theta=0$. Hence we can state the following theorem

Theorem 3.1. A necessary and sufficient condition for a 3-dimensional quasi-Sasakian manifold with constant scalar curvature, to be minimal is that 1.25) holds.

\section{3-dimensional quasi-Sasakian manifolds with $\mathrm{R}(\mathrm{X}, \mathrm{Y}) . \mathrm{S}=\mathbf{0}$}

It is known that for a conformally flat Riemannian manifold

$$
\begin{aligned}
R(X, Y) Z= & \{S(Y, Z) X-S(X, Z) Y+g(Y, Z) Q X-g(X, Z) Q Y\} \\
& -\frac{r}{2}\{g(Y, Z) X-g(X, Z) Y\} .
\end{aligned}
$$

Now let

$$
R(X, Y) . S=0
$$

where $\mathrm{R}(\mathrm{X}, \mathrm{Y})$ is considered as derivation of the tensor algebra at each point of the manifold for tangent vectors $\mathrm{X}, \mathrm{Y}$ and $\mathrm{S}$ is the non-zero Ricci tensor such that

$$
g(Q X, Y)=S(X, Y)
$$

where $\mathrm{Q}$ is the symmetric endomorphosm of the tangent space of M. From 1.27) we have

$$
S(R(X, Y) Z, W)+S(Z, R(X, Y) W)=0 .
$$

Using 1.26) in 1.29) and taking $\mathrm{Y}=\mathrm{Z}$ we have

$$
\begin{aligned}
& g(Z, Z) S(Q X, W)-g(X, Z) S(Q Z, W)+g(Z, W) S(Z, Q X) \\
& -g(X, W) S(Z, Q Z)-\frac{r}{2}\{g(Z, Z) S(X, W)-g(X, Z) S(Z, W) \\
& +g(Z, W) S(Z, X)-g(X, W) S(Z, Z)\}=0 .
\end{aligned}
$$

Let us put $\mathrm{Z}=e_{i}$ where $\left\{e_{i}: \mathrm{i}=1,2,3\right\}$ is the set of orthonormal basis of tangent space at each point of $\mathrm{M}$ and summing for $\mathrm{i}=1,2,3$ we get,

$$
3 S(Q X, W)-g(Q X, Q W)-\frac{r}{2}\{4 g(Q X, W)-g(Q W, X)-r g(X, W)\}=0 .
$$


Let $\lambda$ be any eigen value of the endomorphism $\mathrm{Q}$ corresponding to the eigen vector $\mathrm{X}$. Then

$$
Q X=\lambda X
$$

Using 1.32) in 1.31) and applying 1.28) we get

$$
2 \lambda^{2} g(X, W)-\frac{r}{2}(3 \lambda-r) g(X, W)=0 .
$$

That is,

$$
\lambda^{2}-\frac{3 r}{4} \lambda+\frac{r^{2}}{2}=0
$$

We denote two roots of 1.33) by $\lambda_{1}$ and $\lambda_{2}$. We can write

$$
r=m \lambda_{1}+(3-m) \lambda_{2}
$$

as $\mathrm{r}$ is the trace of $\mathrm{Q}$ and $\mathrm{m}$ is a positive integer which is the multiplicity of $\lambda_{1}$ and hence the multiplicity of $\lambda_{2}$ must be $(3-m)$. From 1.33$)$ we write

$$
\lambda_{1}+\lambda_{2}=\frac{3 r}{4}, \quad \lambda_{1} \cdot \lambda_{2}=\frac{r^{2}}{2} .
$$

Solving 1.34) and 1.35)

and

$$
\lambda_{1}=\frac{3 m-5}{(2 m-3) \cdot 4} r
$$

$$
\lambda_{2}=\frac{3 m-4}{(2 m-3) \cdot 4} r
$$

Thus we state

Theorem 4.1. If a three dimensional quasi-Sasakian manifold with constant scalar curvature satifies $\mathrm{R}(\mathrm{X}, \mathrm{Y}) . \mathrm{S}=0$, then, the symmetric endomorphism $\mathrm{Q}$ of the tangent space corresponding to the Ricci tensor $\mathrm{S}$ has two different non zero eigen values.

\section{References}

[1] D. E. Blair, The theory of quasi-Sasakian structures, J. Differential Geometry 1(1967), 331345 .

[2] D. E. Blair,Contact manifolds in Riemannian Geometry Lecture notes in Math. vol. 509, Springer-Verlag 1976.

[3] B. Y. Chen, Geometry of Submanifolds, Marcel Dekker, New York, 1973.

[4] H. Levi, Symmetric tensors of the second order whose covariant derivative vanishes, Ann. of Math. 27(1926), 91-98. 
[5] Z. Olszak, Normal almost contact metric manifolds of dimension three, Ann. Pol. Math. 47(1986), 41-50.

[6] Z. Olszak, On three-dimensional conformally flat quasi-Sasakian manifolds, Periodica Mathematica Hungarica, 33(2), 1996, 105-113.

[7] R. Sharma, Second order parallel tensor in real and complex space forms, Inter. J. Math. Sci. 12(1989), 787-790. 